\title{
Coulomb and strong interactions for Bose-Einstein correlations
}

\author{
T. Osada ${ }^{1}$, S. Sano ${ }^{2}$ and M. Biyajima ${ }^{2}$ \\ ${ }^{1}$ Department of Physics, Tohoku University, Sendai 980, Japan \\ ${ }^{2}$ Department of Physics, Faculty of Science, \\ Shinshu University, Matsumoto 390, Japan \\ September 3, 2018
}

\begin{abstract}
We present an analytical formula for the Bose-Einstein correlations (BEC) which includes effects of both Coulomb and strong final state interactions (FSI). It was obtained by using Coulomb wave function together with the scattering partial wave amplitude of the strong interactions describing data on the $s$-wave phase shift. We have proved numerically that this method is equivalent to solving Schrödinger equation with Coulomb and the $s$-wave strong interaction potentials. As an application we have analysed, using our formula which includes the degree of coherence and the long range correlation, the data for $e^{+} e^{-}$annihilations.

We have found that the degree of coherence present in our formula approaches approximately unity whereas the long range correlation parameter becomes approximately zero. These results suggest that the physical meanings of the fractional degree of coherence and the long range correlation observed in various other analyses can most probably be attributed to FSI.
\end{abstract}




\section{Introduction}

Very recently several analytical expressions for the final state interaction (FSI) corrections to Bose-Einstein correlations (BEC) due to Coulomb interaction have been presented in 11, 2] (see also [3, 4]). On the other hand it is also well known that there exists FSI due to the strong interactions between identical pions [5, 6, 7]. In order to treat both the FSI of the Coulomb type and those emerging from the strong interactions at the same time, one has to solve Schrödinger equation with the corresponding Coulomb and strong potentials. Such study has been made by Pratt et al. [8] ${ }^{1}$. However, in approach of [8] it is difficult to elucidate the physical meaning of the degree of coherence parameter introduced in various analyses of data on the BEC (see below).

In this paper we consider this problem using different approach from that of 8 . Our aim will be to obtain an analytical formula for the BEC which would include also FSI caused by both Coulomb and strong interactions. Comparing our results with those of [8] we prove numerically that both methods are equivalent. The data of the BEC in $e^{+} e^{-}$annihilation by TPC, AMY, OPAL ALEPH and DELPHI Collaborations 20, 21, 22, 23, 24 are then analysed using our formula. Finally concluding remarks are presented.

\section{Phase shift and wave function of identical $\pi-\pi$ scat- tering}

Let us first consider the Coulomb wave function of the identical $\pi$ - $\pi$ scattering with momenta $p_{1}$ and $p_{2}$ [12, 13:

$$
\Psi_{\mathrm{C}}(\mathbf{k}, \mathbf{r})=\Gamma(1+i \eta) e^{-\pi \eta / 2} e^{i \mathrm{k} \cdot \mathrm{r}} F(-i \eta ; 1 ; i k r(1-\cos \theta)),
$$

\footnotetext{
${ }^{1}$ Study exploiting WKB method is presented in [9]. See also [10] and 11, where $p p$ correlations were studied.
} 


$$
=\sum_{l=0}^{\infty}(2 l+1) i^{l} e^{i \eta_{l}} R_{l}(k r) P_{l}(\cos \theta)
$$

Here $F$ denotes the confluent hypergeometric function and

$$
\begin{aligned}
2 k & =Q=p_{1}-p_{2}, \\
\eta & =\frac{m \alpha}{Q}, \\
\eta_{l} & =\arg \Gamma(l+1+i \eta), \\
R_{l}(k r) & =e^{-\pi \eta / 2} \frac{|\Gamma(l+1+i \eta)|}{(2 l+1) !}(2 k r)^{l} e^{i k r} F(l+1+i \eta ; 2 l+2 ;-2 i k r) .
\end{aligned}
$$

In numerical calculations of the hypergeometric function appearing in (1) wild (oscillating) behavior emerges when $k r$ becomes large. To avoid the computational problem in this asymptotic region, we use there the following asymptotic formula of the Coulomb wave function:

$$
\begin{aligned}
\Psi_{\mathrm{C}}^{\mathrm{asym}}(\mathbf{k}, \mathbf{r})= & \exp \{i(k z+\eta \ln (k(r-z)))\}\left[1+\frac{\eta^{2}}{i k(r-z)}\right] \\
& +f(\theta) \frac{\exp \{i(k r-\eta \ln (2 k r))\}}{r},
\end{aligned}
$$

where $z=r \cos \theta$ and the scattering amplitude $f(\theta)$ is given as follows:

$$
f(\theta)=-\frac{\eta}{2 k} \frac{1}{\sin ^{2}(\theta / 2)} \exp \{-2 i \eta \ln \sin (\theta / 2)+2 i \arg \Gamma(1+i \eta)\}
$$

The asymptotic formula for an $s$-wave component of the Coulomb wave function is obtained in the similar way [13]:

$$
\begin{aligned}
\Psi_{\mathrm{C}(\text { s-wave })}^{\text {asym }}(\mathbf{k}, \mathbf{r})= & \exp \left\{i\left(k r-\eta \ln (2 k r)+2 \eta_{0}\right)\right\} \frac{1}{2 i k r}\left[1+\frac{i \eta(1+i \eta)}{2 i k r}\right] \\
& +\exp \{-i(k r-\eta \ln (2 k r))\} \frac{1}{-2 i k r}\left[1+\frac{i \eta(1-i \eta)}{2 i k r}\right] .
\end{aligned}
$$

As the next step let us consider the strong interaction provided in terms of the phase shift in the identical $\pi-\pi$ scattering. The data for the $s$-wave $(I=2)$ phase shift reported in [14 - 19] can be described phenomenologically by the following formula [5]:

$$
\delta_{0}^{(2)}=\frac{1}{2}\left(\frac{a_{0} Q}{1+0.5 Q^{2}}\right),
$$


where parameter $a_{0}\left(-1.5 \leq a_{0} \leq-0.7\left(\mathrm{GeV}^{-1}\right)\right)$ denotes the scattering length.

Finally, using (4), the scattering s-wave function induced by strong interaction is expressed as:

$$
\begin{aligned}
\phi_{\text {st }}(\mathbf{k}, \mathbf{r}) & =f^{0}(\theta) \frac{\exp \{i(k r-\eta \ln (2 k r))\}}{r}, \\
f^{0}(\theta) & =\frac{1}{2 i k} \exp \left(2 i \eta_{0}\right)\left(\exp \left(2 i \delta_{0}^{(2)}\right)-1\right) .
\end{aligned}
$$

It should be noticed here (cf. Fig. 1(a)) that in addition to (5) we need one more $\pi-\pi$ scattering wave function, which would reasonably describe the strong interaction in the small $k r$ region. Of course Coulomb potential affects both regions, cf. an "unknown box" in Fig.1(a). To obtain a wave function suitable for that box we use the following assumption proposed by Bowler [6] 2]: We assume that the wave function given by (5) with a renormalization provided by the square root of the Gamow factor can be interpolated into the internal region. This is attributed to the normalization of the Coulomb wave function which is given by the following factor: $\sqrt{G(k)}=(2 \pi \eta /(\exp (2 \pi \eta)-1))^{1 / 2}$.

For the asymptotic region we use the following expression:

$$
\Psi_{\text {total }}(\mathbf{k}, \mathbf{r})=\Psi_{\mathrm{C}}^{\mathrm{asym}}(\mathbf{k}, \mathbf{r})+\phi_{\mathrm{st}}(\mathbf{k}, \mathbf{r})
$$

whereas for the region described by the exact Coulomb wave function the following wave function is used instead:

$$
\Psi_{\text {total }}(\mathbf{k}, \mathbf{r})=\Psi_{\mathrm{C}}(\mathbf{k}, \mathbf{r})+\sqrt{G(k)} \phi_{\mathrm{st}}(\mathbf{k}, \mathbf{r})
$$

As seen in Fig.1(b), there is smooth connection between both regions. The usefulness of this assumption will show up in Section 4.

\footnotetext{
${ }^{2}$ Cf. also [7] where the plane wave and the data of the s-wave phase shift of identical $\pi$ - $\pi$ scattering were used. In the present calculation we have to use the Coulomb wave function instead of the plane wave.
} 


\section{Formulation of BEC}

To describe a pair of the identical bosons, we have to symmetrize the total wave function in the following way:

$$
A_{12}=\frac{1}{\sqrt{2}}\left[\Psi_{\mathrm{C}}(\mathbf{k}, \mathbf{r})+\Psi_{\mathrm{C}}^{S}(\mathbf{k}, \mathbf{r})+\Phi_{\mathrm{st}}(\mathbf{k}, \mathbf{r})+\Phi_{\mathrm{st}}^{S}(\mathbf{k}, \mathbf{r})\right],
$$

where superscript $S$ denotes the symmetrization of the wave function. The function $\Phi_{\mathrm{st}}(\mathbf{k}, \mathbf{r})$ stands for the wave function induced by strong interactions. Assuming a source function $\rho(r)$ we obtain the following expression for the BEC including the FSI:

$$
\begin{aligned}
N^{( \pm \pm)} / N^{B G}= & \frac{1}{G(k)} \int \rho(r) d^{3} r\left|A_{12}\right|^{2} \\
= & I_{\mathrm{C}}+I_{\mathrm{Cst}}+I_{\mathrm{st}} \\
I_{\mathrm{C}}= & \sum_{m, n=0}^{\infty} \frac{1}{m+n+1} I_{R 1}(2+m+n) A_{1}(n) A_{1}^{*}(m) \\
& \times\left[1+\frac{n ! m !}{(n+m) !}\left(1+\frac{n}{i \eta}\right)\left(1-\frac{m}{i \eta}\right)\right] \\
= & \left(1+\Delta_{1 \mathrm{C}}\right)+\left(E_{2 \mathrm{~B}}+\Delta_{\mathrm{EC}}\right), \\
I_{\mathrm{C} s t}= & 2 \Re\left[\frac{2}{k}(2 k)^{i \eta} \exp \left(-i\left(\eta_{0}+\delta_{0}^{(2)}\right)\right) \sin \delta_{0}^{(2)} \sum_{n=0}^{\infty} I_{\mathrm{R} 2}(1+n) A_{2}(n, 0)\right], \\
I_{\mathrm{st}}= & \frac{2}{k^{2}} I_{\mathrm{R} 1}(0) \sin ^{2} \delta_{0}^{(2)},
\end{aligned}
$$

where

$$
\begin{aligned}
E_{2 \mathrm{~B}} & =\int d^{3} r \rho(r) e^{-\mathrm{iQ} \cdot \mathbf{r}} \\
1+\Delta_{1 \mathrm{C}} & =1+4 \pi \cdot 2 \eta \int \rho r^{2} d r \sum_{n=0}^{\infty} \frac{(-1)^{n} A^{2 n+1}}{(2 n+1) !(2 n+1)(2 n+2)} \\
A_{1}(n) & =\frac{\Gamma(n+i \eta)}{\Gamma(i \eta)} \frac{(-2 i k)^{n}}{(n !)^{2}} \\
A_{2}(n, l) & =\frac{\Gamma(n+l+1+i \eta) \Gamma(2 l+2)}{\Gamma(l+1+i \eta) \Gamma(n+2 l+2)} \frac{(-2 i k)^{n}}{n !} \\
I_{\mathrm{R} 1}(n) & =4 \pi \int d r r^{n} \rho(r), \\
I_{\mathrm{R} 2}(n) & =4 \pi \int d r r^{n+i \eta} \rho(r) .
\end{aligned}
$$

In this paper $N^{( \pm \pm)} / N^{B G}$ stands for the ratio of pairs of identical charged bosons in a single event to those from different events. Whenever the data are corrected by the 
Gamow factor the final formula should also be divided by the Gamow factor. In numerical computations we have to assume explicitly some forms of the source function. In present calculation we use the gaussian source function: $\rho(r)=\left(\frac{1}{\sqrt{2 \pi} \beta}\right)^{3} \exp \left(\frac{-r^{2}}{2 \beta^{2}}\right)$. Therefore the Fourier transform of the source function is given as follows:

$$
E_{2 \mathrm{~B}}=\exp \left(-\beta^{2} Q^{2} / 2\right)
$$

\section{Comparisons of our results with those of Pratt et al.}

Authors of [8] have presented their results for the BEC with interaction ranges $\beta=2 \mathrm{fm}$ and $\beta=20 \mathrm{fm}$ (corresponding to $R$ in their notation) by solving the following Schrödinger equation:

$$
\left[\frac{d^{2}}{d x^{2}}+K^{2}-\frac{L(L+1)}{x^{2}}-\frac{\epsilon}{x}-U(x)\right] \psi_{L}^{I}(x)=0,
$$

where $\mu=m / 2, x=\mu r, K^{2}=2 E / \mu, \epsilon=2 \alpha$ and $U(x)=\left(2 V_{0} / m_{\rho} x\right) \exp \left(-m_{\rho} x / \mu\right)$ for the $s$-wave. The values of parameters: $V_{0}=2.6 \mathrm{GeV}$ and $m_{\rho}=0.77 \mathrm{GeV}$ were used. In Fig. 2 we compare our results with theirs using the same values of parameters $(\beta=2 \mathrm{fm}$ and $\beta=20 \mathrm{fm})$. Because $I_{\mathrm{C}}$ with $\beta=20 \mathrm{fm}$ shows wild oscillations near $50 \mathrm{MeV} / \mathrm{c}$, which are due to the series expansion of the confluent hypergeometric function $(n+m=75)$, in our calculation we have used a method of seamless fitting introduced in [2]. If we use a different constraint, $n+m=50$, we observe a sharp decreasing near $42 \mathrm{MeV} / \mathrm{c}$, all this depends on the parameter $\beta$. The origin of this phenomenon can be attributed to the mathematical property of the confluent hypergeometric functions and the ability of computers. Therefore in the asymptotic region (2) should be used.

As seen in Fig. 2 our results and solutions of the Schrödinger equation [8] are numeri-

cally equivalent to each other except for the behavior near $Q \cong$ a few $\mathrm{MeV}$ which is due to the logarithmic term in (5). It is therefore confirmed that from their potential $\left(V_{0}=2.6\right.$ 
$\mathrm{GeV}$ ) we obtain in the Born approximation the scattering length $a_{0}=-0.8 \sim-0.6 \mathrm{GeV}^{-1}$.

In the actual analyses we have to introduce a cutoff parameter in the small $Q$ region. Since in many cases there are no data, or available data have large error bars due to limits of momentum resolutions, analyses of data do not critically depend of this cutoff parameter.

\section{Analyses of data in $e^{+} e^{-}$annihilation}

As stressed in Sec.1, we want to elucidate the physical meaning of the degree of coherence. The parameter $\lambda$ describing it should be therefore introduced into (9) in the usual way. Moreover, notice that two more parameters: the additional normalization factor $c$ and the long range correlation parameter $\gamma$ are also introduced by hand. Our final formula containing all these parameters is thus given as:

$$
\begin{aligned}
N^{( \pm \pm)} / N^{\mathrm{BG}}(Q=2 k)= & c\left(1+\Delta_{1 \mathrm{C}}+\Delta_{\mathrm{EC}}+I_{\mathrm{Cst}}+I_{\mathrm{st}}\right) \\
& \times\left[1+\lambda \frac{E_{2 \mathrm{~B}}}{1+\Delta_{1 \mathrm{C}}+\Delta_{\mathrm{EC}}+I_{\mathrm{Cst}}+I_{\mathrm{st}}}\right](1+\gamma Q) .
\end{aligned}
$$

It should be noted that the normalization $c$ and an effective degree of coherence, i.e., the denominator of the ratio $E_{2 \mathrm{~B}} /\left(1+\Delta_{1 \mathrm{C}}+\Delta_{\mathrm{EC}}+I_{\mathrm{Cst}}+I_{\mathrm{st}}\right)$, are related to each other.

For the sake of reference we use in our analyses also the conventional formula (i.e., the standard formula without corrections due to the FSI):

$$
N^{( \pm \pm: \text {Standard })} / N^{\mathrm{BG}}(Q=2 k)=c\left[1+\lambda E_{2 \mathrm{~B}}\right](1+\gamma Q) .
$$

We apply our formulae to data for $e^{+} e^{-}$annihilation provided by [20]-24]. Results of our analyses performed by means of (15) and (16) are shown in Fig. 3 and Table I. 3 As seen in Table I, estimated values of the degree of coherence parameter $\lambda$ are systematically larger (approaching unity) when (15) is used than those obtained by the standard formula (16).

\footnotetext{
${ }^{3}$ It is difficult to treat $a_{0}$ as a free parameter in the CERN MINUIT program, due to the limited ability of our computer.
} 
On the other hand, estimated values of the long range correlation parameter $\gamma$ approach approximately zero when (15) is used (except for the result found in present analysis of data by AMY collaboration).

Finally please notice that reported values in [20 - 24 are obtained by the following formula:

$$
N^{( \pm \pm: \text {Empirical. })} / N^{\mathrm{BG}}(Q=2 k)=c\left[1+\lambda \exp \left(-Q^{2} R^{2}\right)\right](1+\gamma Q)
$$

To compare our estimated values with various reported ones, we have to use a relation,

$\beta=\sqrt{2} R$, because of the different method of integrations. In Table I we show therefore the corrected values instead of the values reported by various collaborations. The $\beta$ 's obtained in the analysis by (15) are systematically smaller than the corrected values taken from [20] -24] and the estimated ones obtained in the analysis by the standard formula (16).

\section{Concluding remarks}

We obtain analytic formula (9) for BEC including the Coulombic and strong FSI. It is (numerically) confirmed that our method is equivalent to solving the Schrödinger equation (14).

Combining the seamless fitting method [2] and the CERN MINUIT program in (15) we have analysed data for BEC in $e^{+} e^{-}$annihilations. Our results are significantly different from those obtained by the standard formula (16). Namely, it is found that the degree of coherence parameter $\lambda$ and the long range correlation parameter $\gamma$ approaches approximately unity and zero, respectively (see Table I). Therefore, we conclude that the physical meanings of the fractional degree of coherence parameter $\lambda$ and the long range correlation parameter $\gamma$ as obtained by the standard formula (16) should be attributed to the FSI. Moreover, the values of the source size parameter reported by various collaborations (after using the relation: $\beta=\sqrt{2} R$ ) and obtained by the standard formula (16) 
are systematically larger than values estimated by (15).

Acknowledgements: The authors would like to thank T. Mizoguchi, H. Sagawa, T. Sasakawa, F. Shibata and T. Ueda for their kind correspondences. Numerical computations are partially done by the computer at Bubble Chamber physics Laboratory (Tohoku). This work is partially supported by Japanese Grant-in-Aid for Scientific Research ¿from the Ministry of Education, Science, Sport and Culture (\# 06640383). Finally the authors are also indebted to G. Wilk for his reading the manuscript. 


\section{References}

[1] M. Biyajima, T. Mizoguchi, T. Osada and G. Wilk, Phys. Lett. B353 (1995) 340; see also: M. Biyajima and T. Mizoguchi, SULDP-94-9 (Dec. 1994).

[2] M. Biyajima, T. Mizoguchi, T. Osada and G. Wilk, DPSU-95-4 (July 1995); hepph/9507210. To appear in Phys. Lett. B.

[3] M. G. Bowler, Phys. Lett. B270 (1991) 69.

[4] R. Lednicky, V.L. Lyuboshits, Sov. J. Nucl. Phys. 35 (1982) 770.

[5] M. Suzuki, Phys. Rev. D35 (1987) 3359.

[6] M. G. Bowler, Z. Phys. C39 (1988) 81.

[7] M. Biyajima, T. Mizoguchi and G. Wilk, Z. Phys. C65 (1995) 511.

[8] S. Pratt, T.Csörgö and J. Zimanyi, Phys. Rev. C42 (1990) 2646.

[9] D. Anchishkin and G. Zinojev, Phys. Rev. C51 (1995) R2306.

[10] S. Koonin, Phys. Lett. B70 (1977) 43.

[11] M. Biyajima, Phys. Lett. B132 (1983) 299.

[12] L. I. Schiff, Quantum Mechanics, 2nd Ed. (McGraw-Hill, New York, 1955) p.117.

[13] T. Sasakawa, Scattering Theory, (in Japanese) (Shokabo-book Co., Tokyo, 1991).

[14] W. D. Walker et al., Phys. Rev. Lett. 18 (1970) 630.

[15] E. Colton et al., Phys. Rev. D3 (1971) 2028.

[16] D. Cohen et al., Phys. Rev. D7 (1973) 661.

[17] J. P. Prukop et al., Phys. Rev. D10 (1974) 2055.

[18] M. Baubillier et al., Phys. Lett. 45B (1973) 517. 
[19] W. Hoogland et al., Nucl. Phys. B69 (1974) 266.

[20] H. Aihara et al. (PEP4-TPC Collaboration), Phys. Rev. D31 (1985) 996.

[21] S.K. Choi et al. (AMY Collaboration), Phys. Lett. B355 (1995) 406.

[22] P. D. Acton et al. (OPAL Collaboration), Phys. Lett. B267 (1991) 143.

[23] D. Decamp et al. (ALEPH Collaboration), Z. Phys. C54 (1992) 75.

[24] P. Abreu et al. (DELPHI Collaboration), Phys. Lett. B286 (1992) 201. 


\section{Figure Captions}

Fig. 1. (a) Interrelation between the two wave functions in external and internal regions. (b) Real part of total wave function with $Q=100 \mathrm{MeV} / \mathrm{c}$ and $\theta=\pi / 2$. See (7). Near $250 \mathrm{MeV} / \mathrm{c}$ there is a connecting point.

Fig. 2. Comparisons of our results (solid line) and those of Pratt et al., [8] (dashed line). The scattering length $a_{0}=-0.6 \mathrm{GeV}^{-1}$ is used. This value approximately corresponds to $V_{0}=2.6 \mathrm{GeV}$ in the Born approximation. (a) $\beta=2 \mathrm{fm}$ and (b) $\beta=20 \mathrm{fm}$. Behaviors in small $Q$ region (see inserts) are attributed to the logarithmic term in (5).

Fig. 3. (a) Analysis of data of TPC Collaboration [20] by (15) and (16).

(b) The same as (a) but for data of AMY Collaboration [21]. (The point at the smallest value of $Q$ is neglected in analysis by means of (15).).

(c) The same as (a) but data of OPAL Collaboration [22].

(d) The same as (a) but data of ALEPH Collaboration [23].

(e) The same as (a) but data of DELPHI Collaboration [24].

\section{Table Caption}

Table I. Analyses of data of the BEC by TPC, AMY, OPAL, ALEPH and DELPHI Collaborations; $a_{0}=-1.00 \mathrm{GeV}^{-1}$. The source size parameters obtained by all collaborations

are corrected by $\beta=\sqrt{2} R$. There is no significant difference between those corrected values (denoted by $(*)$ ) and values obtained in present analysis. The AMY collaboration has used the fitting function $R_{\text {mix }}(Q)=C\left(1+f_{\pi}(Q) \lambda \exp \left(-\beta^{2} Q^{2} / 2\right)\right)(1+\gamma Q), f_{\pi}(Q)=$ $0.719-0.070 Q+0.056 Q^{2}-0.020 Q^{3}$. 


\begin{tabular}{|c|c|c|c|c|c|c|}
\hline & & $\beta[\mathrm{fm}]$ & $\lambda$ & $\gamma$ & $c$ & $\chi^{2} / \mathrm{NDF}$ \\
\hline \multicolumn{7}{|l|}{$\mathrm{TPC}$} \\
\hline [20]: & & $0.92 \pm 0.06^{*}$ & $0.61 \pm 0.05$ & - & - & - \\
\hline \multirow[t]{2}{*}{ Our analyses: } & (15) & $0.737 \pm 0.050$ & $1.097 \pm 0.042$ & $-0.000 \pm 0.020$ & $1.002 \pm 0.022$ & $44.2 / 35$ \\
\hline & (16) & $0.912 \pm 0.062$ & $0.611 \pm 0.054$ & $0.083 \pm 0.025$ & $0.881 \pm 0.023$ & $41.0 / 35$ \\
\hline \multicolumn{7}{|l|}{ AMY } \\
\hline [21]: & & $0.823 \pm 0.088^{*}$ & $0.392 \pm 0.041$ & $0.033 \pm 0.041$ & $0.935 \pm 0.016$ & $90.2 / 93$ \\
\hline \multirow[t]{2}{*}{ Our analyses: } & (15) & $0.460 \pm 0.039$ & $0.947 \pm 0.033$ & $-0.078 \pm 0.012$ & $1.203 \pm 0.030$ & $106.8 / 95$ \\
\hline & (16) & $0.854 \pm 0.088$ & $0.286 \pm 0.030$ & $0.030 \pm 0.013$ & $0.956 \pm 0.016$ & $94.1 / 96$ \\
\hline \multicolumn{7}{|l|}{ OPAL } \\
\hline [22]: & & $1.124 \pm 0.021^{*}$ & $0.846 \pm 0.025$ & - & - & $336 / 73$ \\
\hline \multirow[t]{2}{*}{ Our analyses: } & (15) & $1.090 \pm 0.035$ & $1.043 \pm 0.025$ & $0.003 \pm 0.004$ & $0.991 \pm 0.005$ & $124.4 / 74$ \\
\hline & (16) & $1.339 \pm 0.035$ & $0.713 \pm 0.036$ & $0.040 \pm 0.004$ & $0.936 \pm 0.004$ & $118.7 / 74$ \\
\hline \multicolumn{7}{|l|}{ ALEPH } \\
\hline [23]: & & $1.14 \pm 0.06^{*}$ & $0.48 \pm 0.03$ & $0.02 \pm 0.01$ & $0.97 \pm 0.01$ & $77 / 70$ \\
\hline \multirow[t]{2}{*}{ Our analyses: } & (15) & $0.917 \pm 0.037$ & $1.070 \pm 0.024$ & $-0.016 \pm 0.008$ & $1.032 \pm 0.011$ & $89.0 / 69$ \\
\hline & (16) & $1.128 \pm 0.037$ & $0.630 \pm 0.030$ & $0.024 \pm 0.009$ & $0.964 \pm 0.011$ & $87.3 / 69$ \\
\hline \multicolumn{7}{|l|}{ DELPHI } \\
\hline [24]: & & $1.16 \pm 0.04^{*}$ & $0.45 \pm 0.02$ & - & - & $89 / 73$ \\
\hline \multirow[t]{2}{*}{ Our analyses: } & (15) & $0.871 \pm 0.027$ & $0.946 \pm 0.016$ & $-0.001 \pm 0.006$ & $1.020 \pm 0.008$ & $90.3 / 73$ \\
\hline & (16) & $1.170 \pm 0.039$ & $0.451 \pm 0.020$ & $0.033 \pm 0.007$ & $0.963 \pm 0.008$ & $89.1 / 73$ \\
\hline
\end{tabular}

Table I 


\begin{tabular}{|c|c|c|}
\hline Region & $\begin{array}{l}\text { Region described } \\
\text { by exact } \\
\text { Coulomb wave function }\end{array}$ & $\begin{array}{l}\text { Asymptotic } \\
\text { region }\end{array}$ \\
\hline $\begin{array}{l}\text { Coulomb } \\
\text { wave } \\
\text { fuction } \\
\qquad \Psi_{\mathrm{C}}\end{array}$ & $\begin{array}{l}\text { Exact } \\
\Psi_{\mathrm{C}}\end{array}$ & $\begin{array}{l}\text { Asymptotic } \\
\qquad \Psi_{\mathrm{C}}^{\text {asym }}\end{array}$ \\
\hline $\begin{array}{l}\text { Scattering } \\
\text { wave } \\
\text { function } \\
\qquad \Phi_{\text {st }}\end{array}$ & 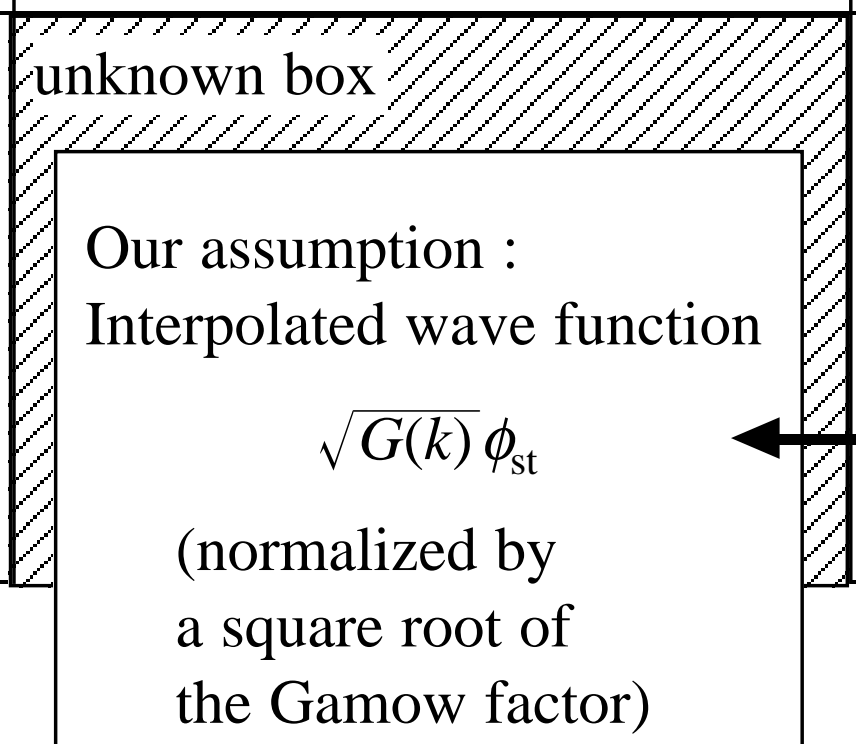 & $\begin{array}{l}\text { Scattering } \\
\text { s-wave } \\
\text { function } \\
\qquad \phi_{\text {st }}\end{array}$ \\
\hline
\end{tabular}

Fig.1(a) 


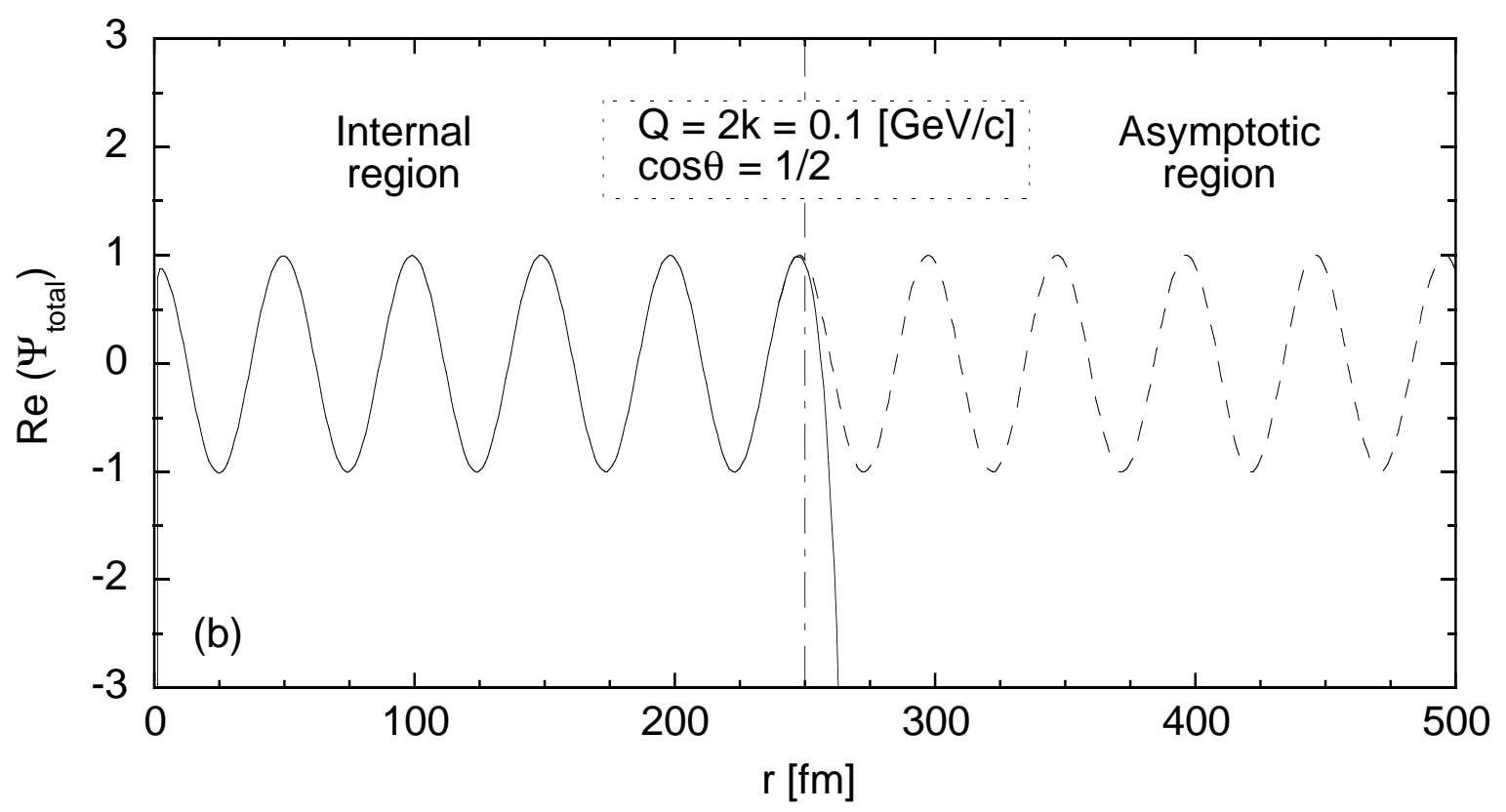

Fig.1(b) 


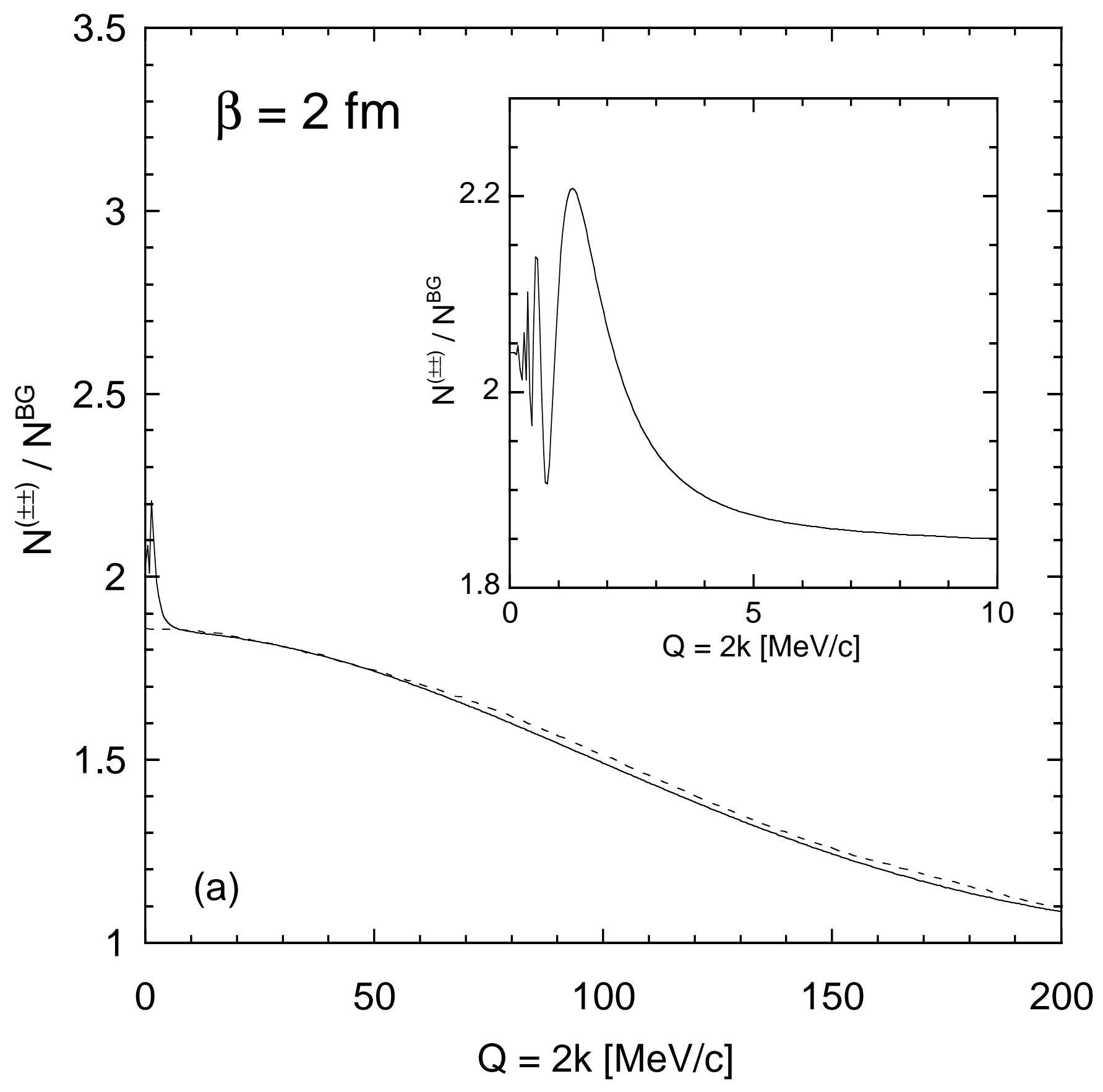

Fig.2(a) 


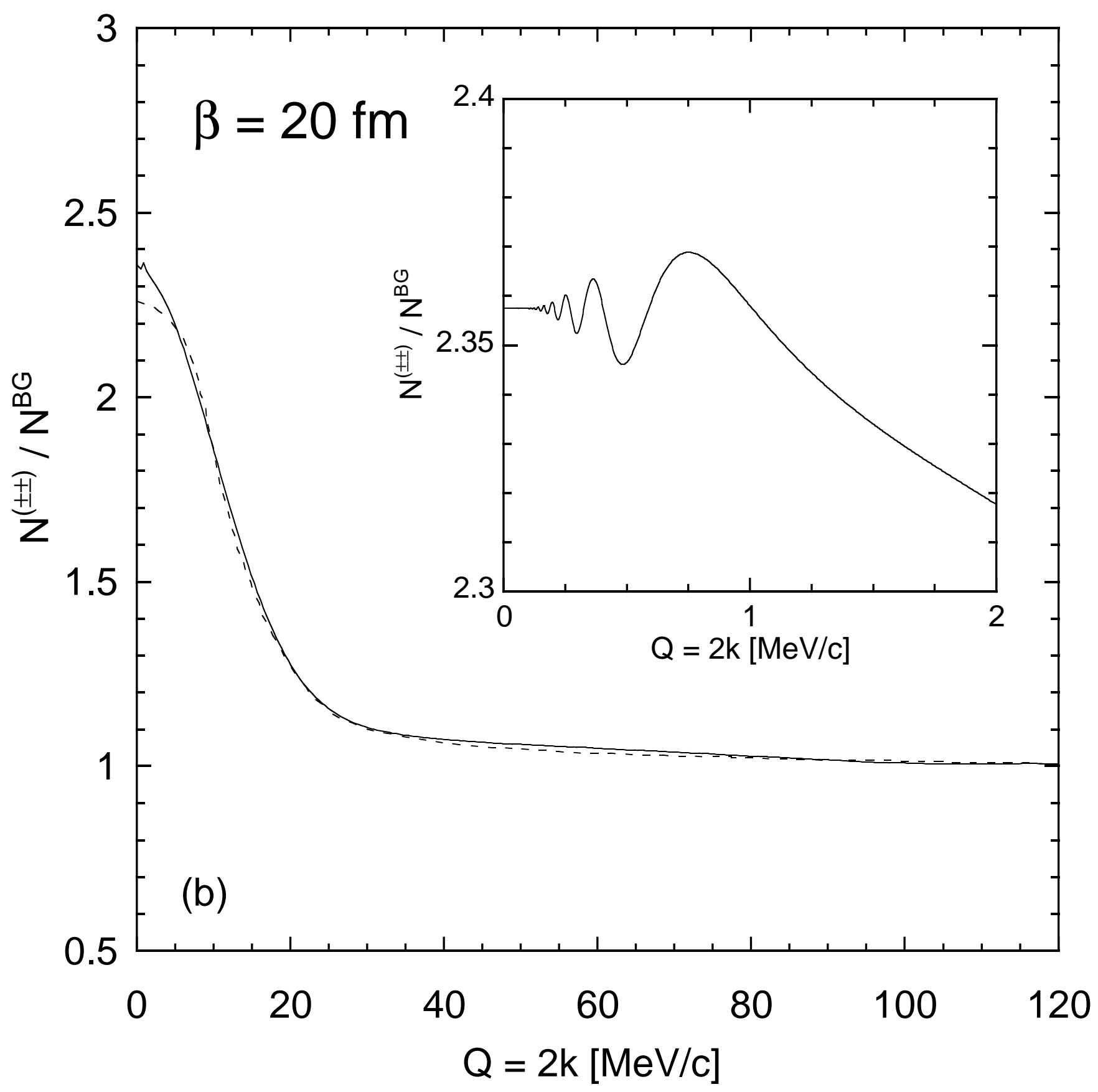

Fig.2(b) 


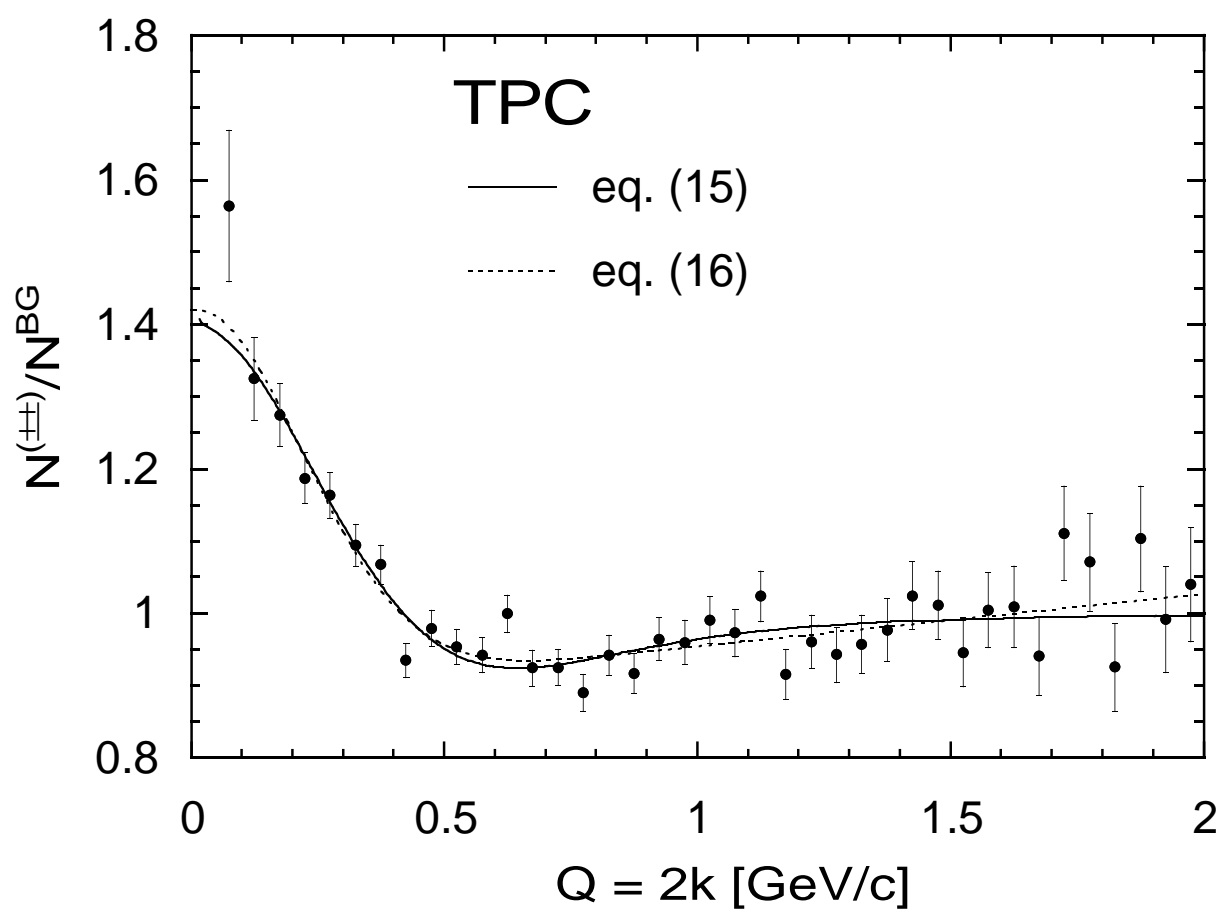

Fig.3(a) 


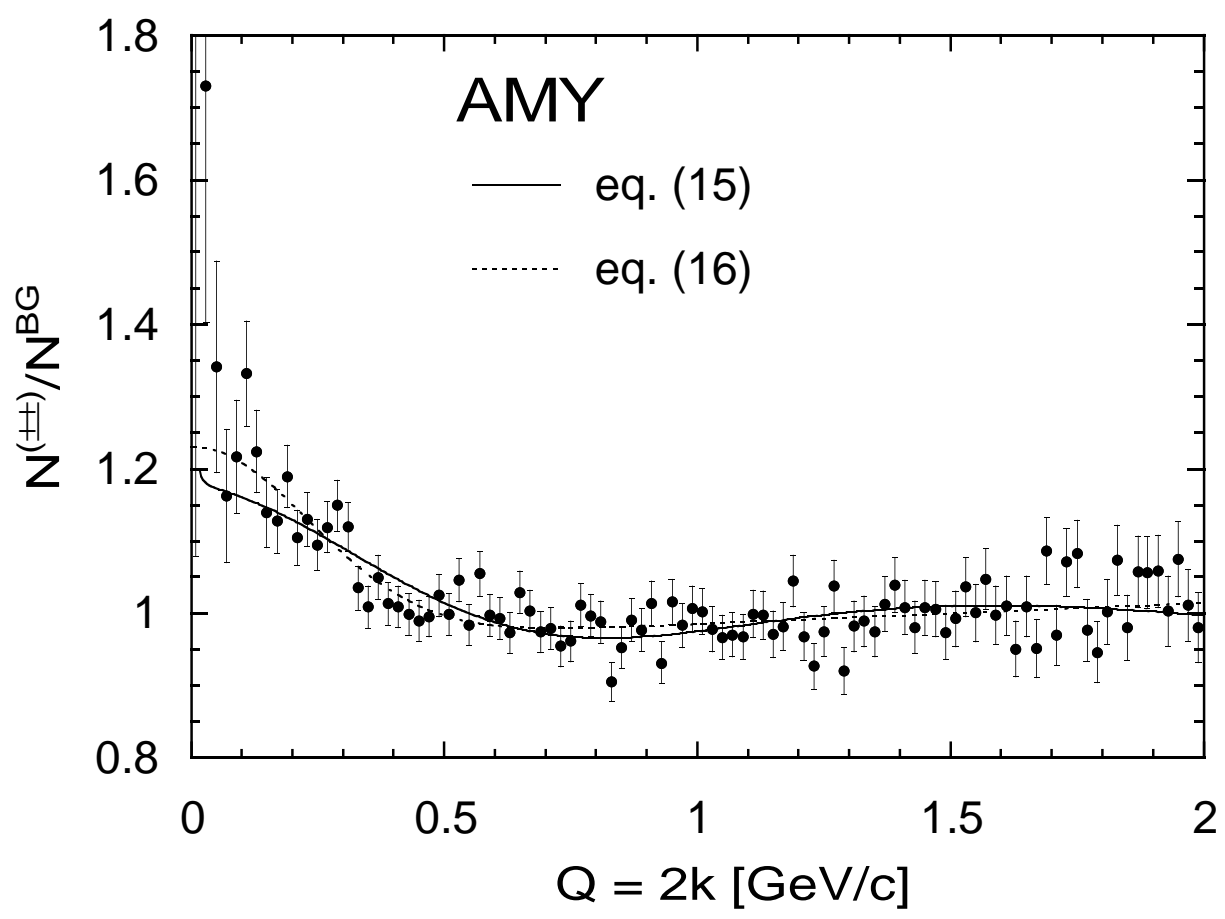

Fig.3(b) 


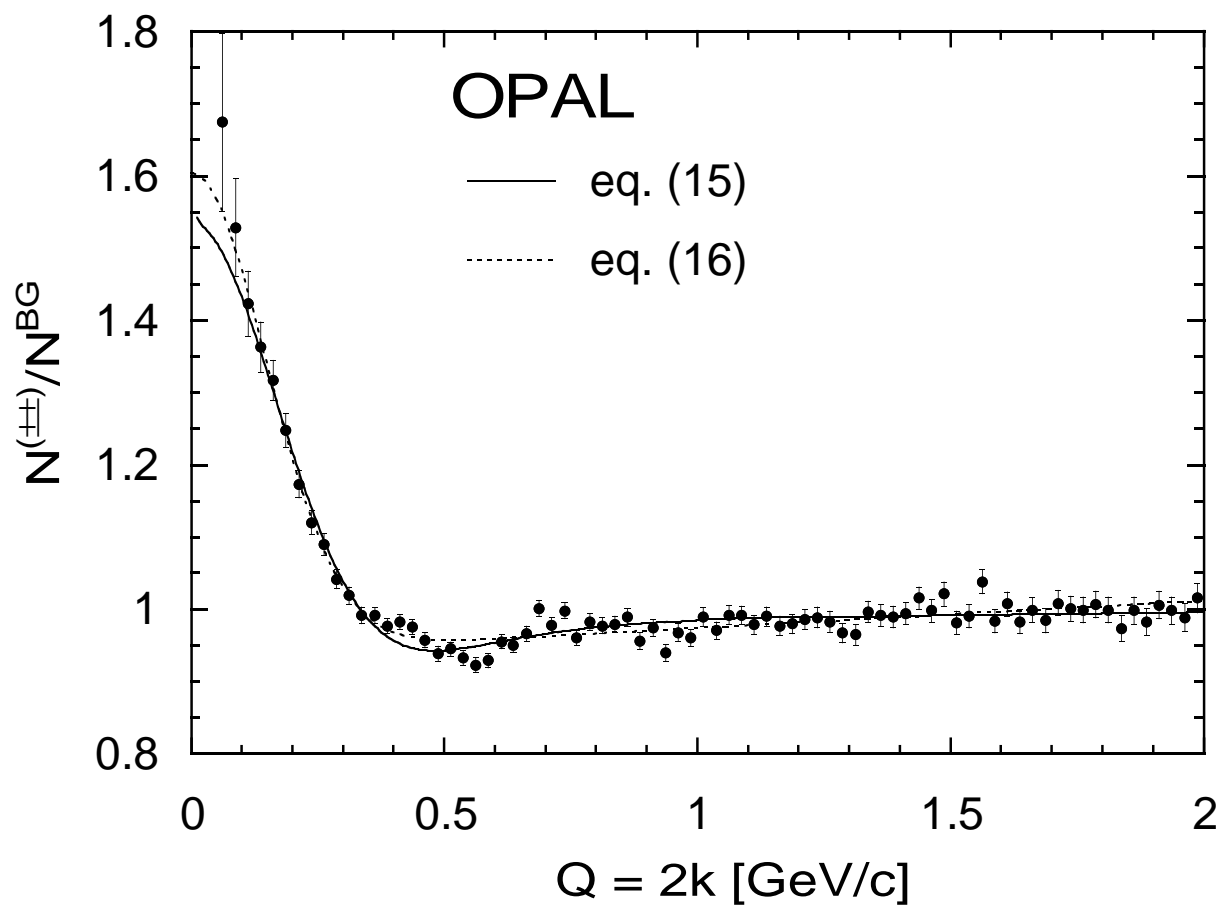

Fig.3(c) 


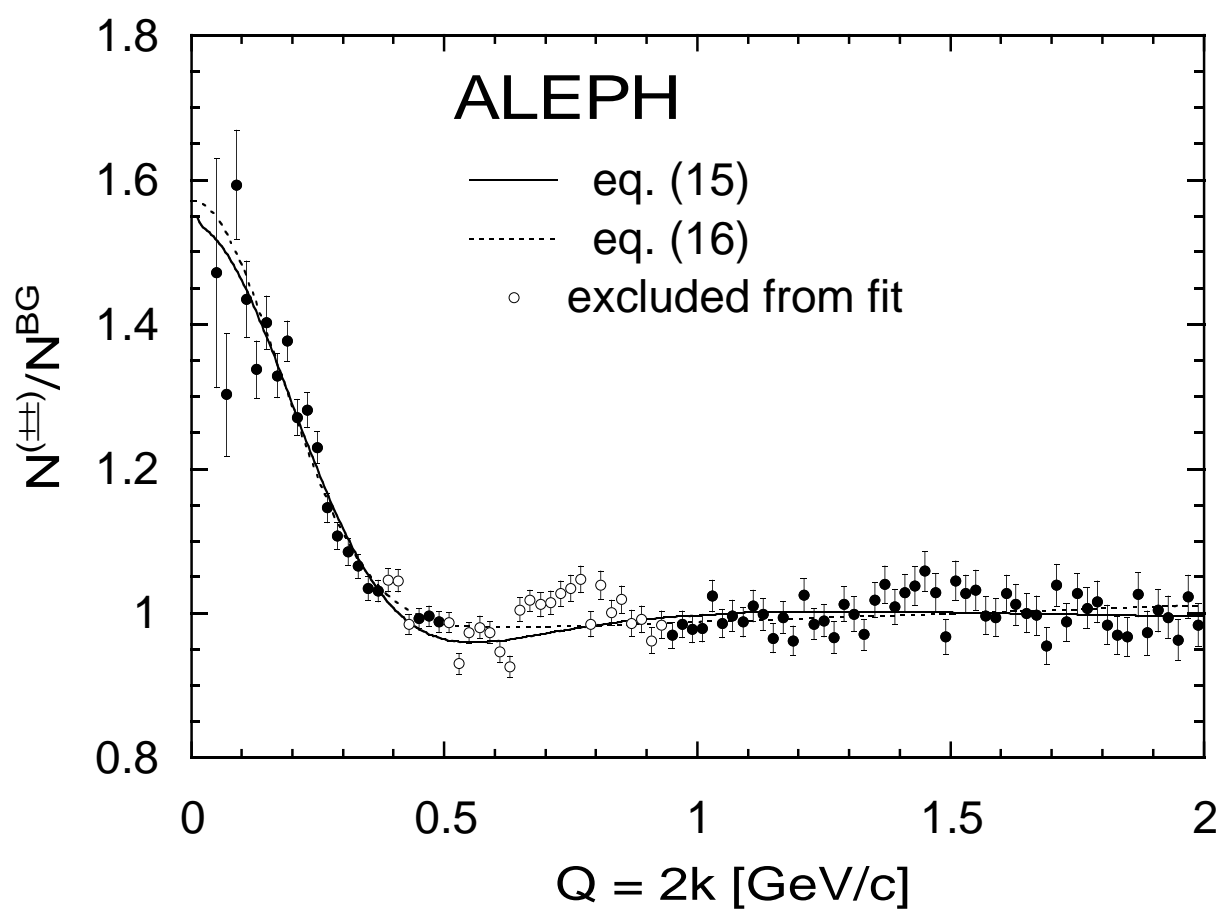

Fig.3(d) 


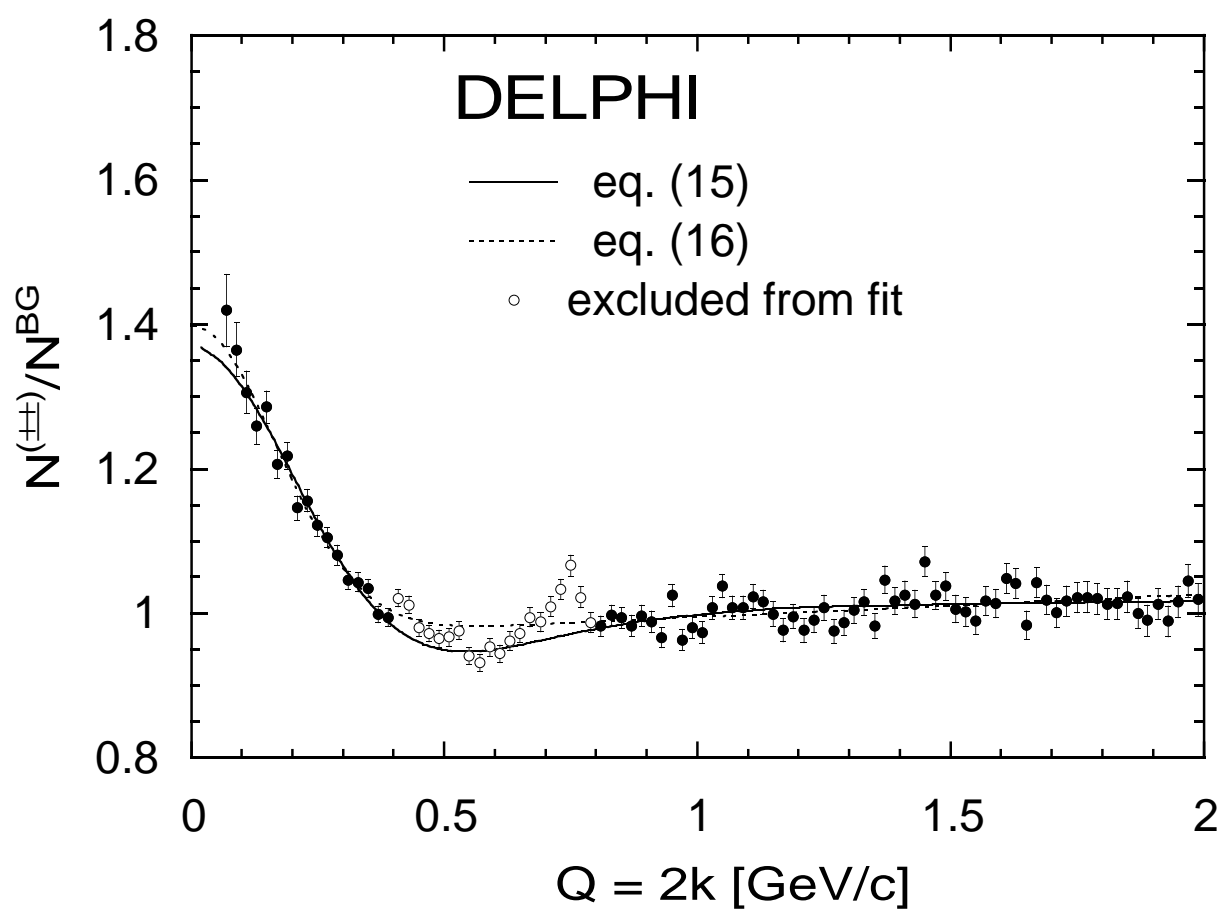

Fig.3(e) 